\title{
Effects of Different Forms of Maternal Hypertension During Pregnancy On Neonatal Outcomes
}

\author{
S.. Osman', S. Khan', A. Soliman', H. Salama², H. Al-Rifai', M. Al-Qubasi, \\ T. Olukade ${ }^{2}, \underline{H . \text { Mahjoob }^{1}}$ \\ 1-Department of General Pediatrics, Hamad Medical Corporation, Qatar \\ 2-Department of Neonatology, Hamad Medical Corporation, Qatar
}

\section{Background and Aims}

Hypertension in pregnancy is not uncommon, and its neonatal outcomes are variable among different populations.

\section{Method}

We conducted a research using data from the birth registry obtained from our Maternity hospital in Qatar to evaluate neonatal outcomes of pregnant women with hypertension. Hypertension was defined based on ISSHP into: Essential hypertension (EH), Gestational hypertension (GH) and Preeclampsia / eclampsia (PEH).

\section{Results}

Of the 14011 pregnant women screened during 2017 with singleton delivery, 181 had PEH (1.3\%), $253 \mathrm{had} \mathrm{GH}(1.8 \%)$ and $117 \mathrm{had}(0.8 \%) \mathrm{EH}$ with total prevalence of hypertension being 3.9\%.Women with dysglycemia showed higher prevalence of essential hypertension compared to their normoglycemic counterparts $(p<0.001)$. All the three forms of hypertension, had higher rates of $C$ section delivery compared to the normotensive group $p<0.001$. The incidence of LBW $(<2500$ grams) term babies was higher in women with hypertension (PEH 14.4\%, GH 8\%, EH 6.4\%) compared to $3 \%$ among normotensive women $(p<0.001)$. Premature delivery was significantly more in PEH, GH and EH (38.7\%, 20.6\% and $19.7 \%$ respectively) compared to control (6.6\%). Newborns of women with PEH, GH and EH were more likely to need NICU admission $\mathbf{4 0 . 6 \% , 2 7 . 9 \%}$ and $21.6 \%$ respectively) compared to normotensive group (12.7\%) $p<0.001$. Respiratory distress and low Apgar score was commoner in newborns of hypertensive mothers ( $p$ value $<0.001$ and 0.002 respectively). Still birth and neonatal mortality did not differ.

\section{Conclusion}

Maternal hypertension is an important cause of neonatal morbidity including higher frequency of respiratory distress, LBW, low Apgar score, prematurity and NICU admissions.

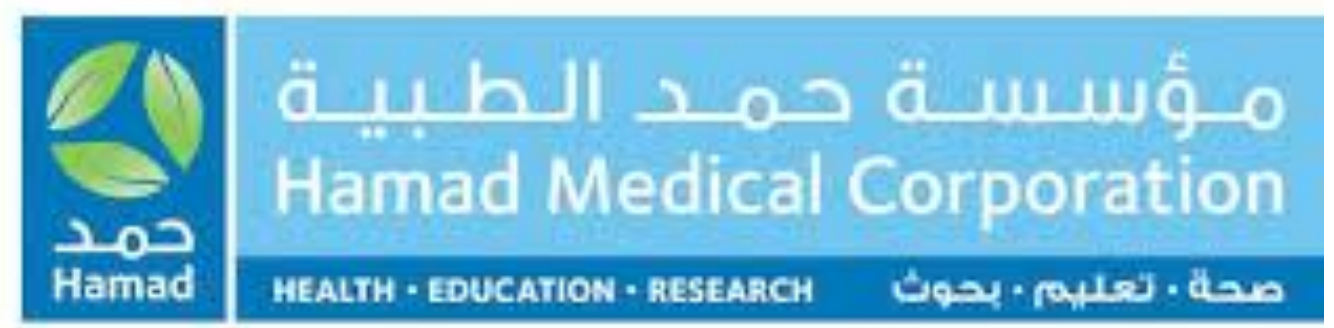

